\title{
Nutritional strategies in managing postmeal glucose for type 2 diabetes: a narrative review
}

\begin{abstract}
Medical Nutrition Therapy (MNT) plays an essential role in overall glycemic management. Less focus is given on managing postmeal hyperglycemia despite the facts that, it is a common feature of Type 2 Diabetes (T2D). The purpose of this narrative review is to provide a comprehensive understanding of the existing literature on the nutritional approaches to improve postmeal hyperglycemia in patients with T2D. We searched multiple databases for the studies examining the nutritional approaches to manage postmeal glucose in patients with T2D. We included studies that involve human trials that were published in English for the past 10 years. Our review of the current literature indicates that the postmeal hyperglycemia can be improved with four nutritional approaches. These approaches include (i) utilizing the appropriate amount and selecting the right type of carbohydrates, (ii) using specific types of dietary protein, (iii) manipulating the meal timing and orders and (iv) others (promoting postmeal physical activity, incorporating diabetes-specific formula and certain functional foods). The potential mechanisms underlying these approaches are discussed and the identified gaps warranted further research. This array of nutritional strategies provide a set of options for healthcare professionals to facilitate patients with T2D in achieving the optimal level of postmeal glucose.
\end{abstract}

Keyword: Diet; Medical nutrition therapy; Postmeal glucose; Postprandial glucose; Type 2 diabetes 